\title{
Lung Function Abnormalities among Tannery Workers in Vellore District
}

\author{
Adikesavan B. ${ }^{1}$, Sangeetha P. ${ }^{1}$ \\ ${ }^{1}$ Associate Professor, Department of Physiology, Govt.Vellore Medical College, Vellore-11
}

\begin{abstract}
Vellore and its allied areas have a high concentration of tanneries. There is a very large population engaged in the different sections of the tanneries. They are constantly exposed to leather tanning chemicals like chromium, $\mathrm{HCL}, \mathrm{H}_{2} \mathrm{SO}_{4}$, alum etc. The constant exposure to these chemicals makes the workers susceptible to many lung function abnormalities. More over the contact with these chemicals can cause skin diseases, nasal cancer, ocular abnormalities and respiratory diseases. Duration of exposure is a major criterion for the reduction in lung function. It is a cross sectional study involving 39 male subjects aged between 18-60 years working in different sections of the tanneries in Vellore district as study group and 25 male subjects of the same age group and socio economic status and who do not have any previous exposure to leather tanning chemicals as control group . Lung function testing was done using Eagles's global spirometer. Our study shows moderate restriction and mild obstruction in study group when compared to normal subjects and also shows progressive decrement in FVC and FEFmax with their age and duration of occupation.
\end{abstract}

Keywords: Tannery workers, PFT, FVC, FEF max.

\section{Introduction}

In Tamil Nadu, Vellore district was found to have more number of tannery factories. Vellore district is the topmost suppliers about $37 \%$ of finished leather around the country. ${ }^{(1)}$ Vellore and its allied areas have a high concentration of tanneries. There is a very large population engaged in the different sections of the tanneries. They are constantly exposed to leather tanning chemicals like chromium, Hydrochloric acid ( $\mathrm{HCl})$, Hydrogen sulphate $\left(\mathrm{H}_{2} \mathrm{SO}_{4}\right)$, alum etc. Constant exposure to environmental chromium this can affect lung airways and parenchyma. A study among chrome tannery

\section{Corresponding Author:}

\section{Adikesavan B.}

Associate Professor, Department of Physiology, Govt. Vellore Medical College, Vellore-632011

Mobile: 9443686517

e-mail: balajiadhi@yahoo.com workers showed that there was significant prevalence of cancer not only of the lungs but also pancreas, bladder, kidney, testes, nasal cavity, lymphoma etc. ${ }^{(2)}$ Another risk is exposure to chromium as it enters the body through inhalation, ingestion leading to the risk of nasal septal perforations ${ }^{(3)}$ and even direct cutaneous contact with chromium can cause severe eczema. ${ }^{(4)}$ The exposure to these chemicals makes the workers susceptible to many lung function abnormalities. More over the contact with these chemicals can cause skin diseases, nasal cancer, ocular abnormalities and respiratory diseases. Duration of exposure is a major criterion for the reduction in lung function. Benzene based dyes and formaldehyde are the chemicals used in tanning process are considered to be carcinogenic. ${ }^{(5)}$ According to the International Agency for Research on Cancer (IARC), showed that there is evidence of carcinogenicity in humans who work in leather tanning and processing whereas the production process involves more exposure to numerous chemicals. ${ }^{(6)}$ Leather tanners can be also exposed to pesticide which can cause prostate cancer. ${ }^{(7)}$ 
Table 1: Shows here the chemicals used in the process of tanning and its purpose (8) Kanpur-occupational

\begin{tabular}{|c|l|l|l|}
\hline S.No & Processes & Chemical Used & Purpose \\
\hline 1 & Preparation for hide tanning & $\begin{array}{l}\text { DDT, Zincchloride, phenols, formaldehyde, } \\
\text { arsenic }\end{array}$ & Defestationand disinfection \\
\hline 2 & Tanning process & $\begin{array}{l}\text { Calcium hydroxide, sulphide, benzene, ethanol, } \\
\text { H2SO4 }\end{array}$ & $\begin{array}{l}\text { Removal of epidermis and } \\
\text { subcutaneous layer. }\end{array}$ \\
\hline 3 & $\begin{array}{l}\text { Finishing-Casein finishing \& } \\
\text { Nitrocellulose finishing }\end{array}$ & Formaldehyde as fixer, anilines and resins & $\begin{array}{l}\text { Coloring, softness, brightness, } \\
\text { elasticity }\end{array}$ \\
\hline
\end{tabular}

Hence we choose to do pulmonary function tests in tannery workers who come to the chest clinic with respiratory symptoms to our hospital.

\section{Materials and Methodology}

This study was done in the Vellore district involving the tannery workers and lung function tests were done in the department of Physiology, Government Vellore medical college (GVMC). This cross sectional study involved 39 male subjects aged between 18-60 years working in different sections of the tanneries in Vellore district as study group were the patients attending the chest clinic outpatient department (OPD) in our hospital. The control group 25 male subjects of the same age group and socio economic status and also who do not have any previous exposure to leather tanning chemicals were the patients attending the medicine OPD in our hospital. More importance was given while taking history where mainly focussed on personal and occupational history. The Personal history includes the name, age, height, weight, BMI, smoking history, alcohol consumption, history of previous respiratory illnesses, surgeries etc. The Occupational history included occupational duration, type of work, toxicants they work with and the mode of contact with the chemicals. The medicine and chest clinic were intimated earlier about the study and contact number of the principal investigator (PI) were also given.So before sending the patient to the physiology department for doing PFT, the PI was also intimated. The study was proceeded after getting consent from the both the groups.

Lung function testing was done using "EAGLES'S GLOBAL SPIROMETER" in the department of
Physiology and the following parameters were studied - FVC, FEV ${ }_{1}$, FEV1/FVC, FEF 25-75\%, FEF max and MVV.

Forced vital capacity/Timed vital capacityMaximum volume of air that can be breathed out forcefully and rapidly after a maximum inspiration.

$\mathrm{FEV}_{1}$ (Forced vital capacity in 1 second)- Volume of FVC expired in one second of exhalation.

FEF $25-75 \%$-The maximum flow during the middle third of the total expired volume which is expressed as forced expiratory flow at $25 \%$ to $75 \%$ of lung volumes.

MVV-(Maximum voluntary ventilation)-also called maximum breathing capacity where the maximum volume of gas can be breathed per minute by maximal voluntary effort.

\section{Results}

The comparison of the mean values of the anthropometric measure between the study and the control group was done. But there was not much significant because both the groups were of the same age and socio-economic status. The table 2 below shows the mean values of FVC, FEV1/FVC, FEF25-7\%, FEF max and MVV when compared between the study and the control group showed significant reduction in all the parameters among the study group especially in FVC and MVV. So there is $22 \%$ reduction in FVC with $\mathrm{p}$ value of $0.019,23 \%$ reduction in FEF $25-75 \%$ with $p$ value of $0.001,13 \%$ reductionin FEF max with $p$ value of 0.00021 and $85 \%$ reduction in MVV. 
Table 2: Shows the comparison between the control and study group of the lung parameters assessed

\begin{tabular}{|l|c|c|c|}
\hline Parameters & Control (Mean) & Study (Mean) & P-value \\
\hline FVC & 3.51133 & 2.7128 & $>0.019581$ \\
\hline FEV1 & 3.25933 & 2.616 & \\
\hline FEV1/FVC & 92.45867 & 95.4872 & $>0.009930$ \\
\hline FEF25-75\% & 4.55267 & 3.4964 & $>0.000029$ \\
\hline FEFmax & 8.606 & 7.4772 & \\
\hline MVV & 127.81867 & 109.3488 & \\
\hline
\end{tabular}

The reduction in FEF $25 \%$, FEF 75\%, FEF 50\% among the study group when compared to the control group and also the study group showed 13\% reduction in FEF max is shown in Fig 1 as bar graph representation below.

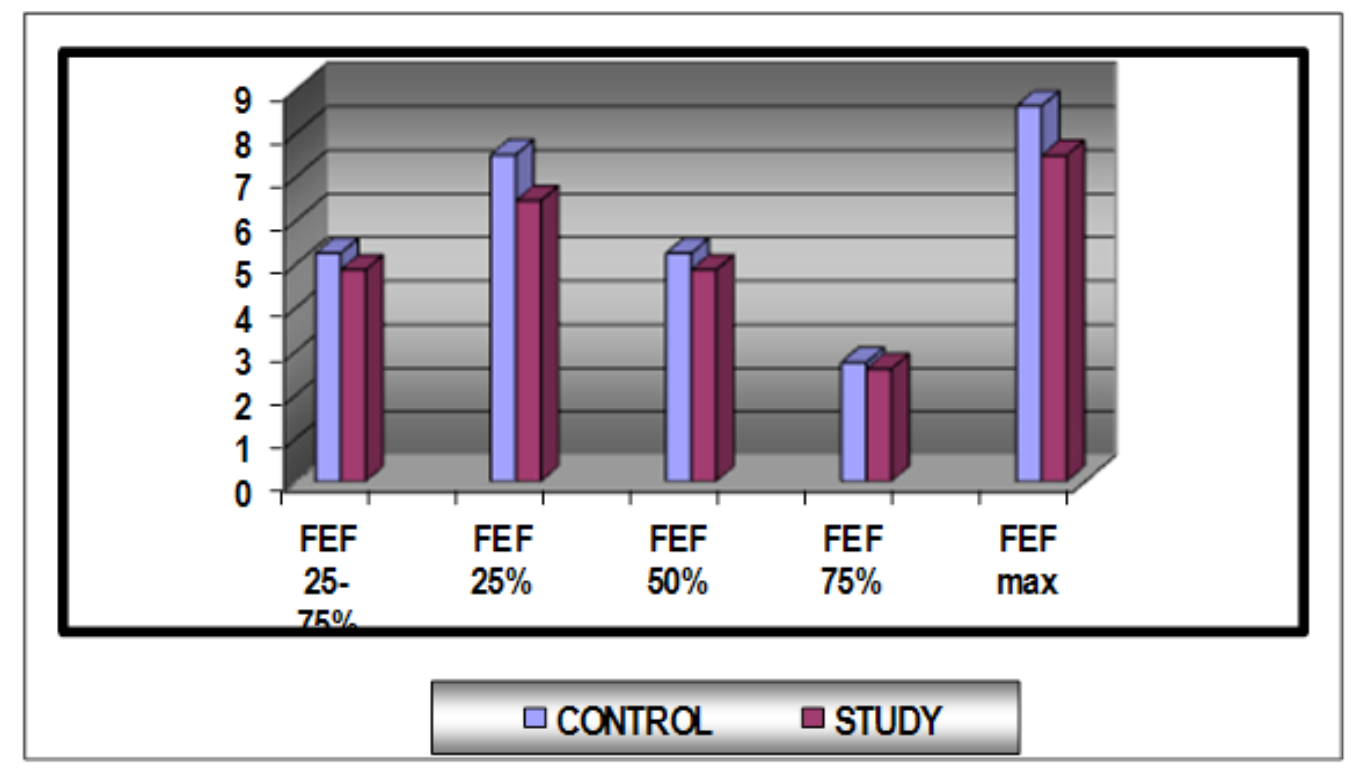

Fig 1: Shows the bar graph comparison of Various FEF values between the study and control groups

The Co-relation of FVC with duration of occupational years of exposure showed an inverse relationship between them, that is the more was the occupational duration, the less was the value of FVC has been shown below in fig 2.

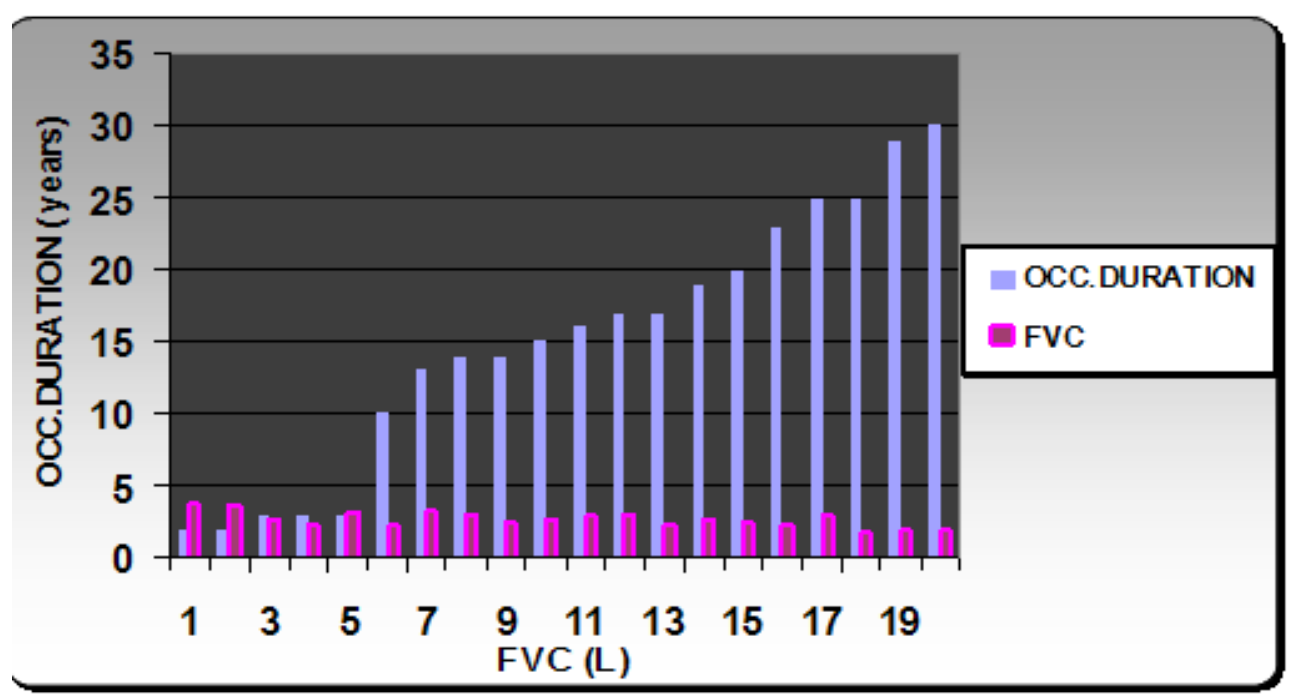

Fig 2: Shows the bar graph comparison of FVC values with the occupational years 
Fig 3 below shows that the correlation between MVV and occupational duration also showed an inverse relationship between them. The MVV decreases as the occupational duration increases.

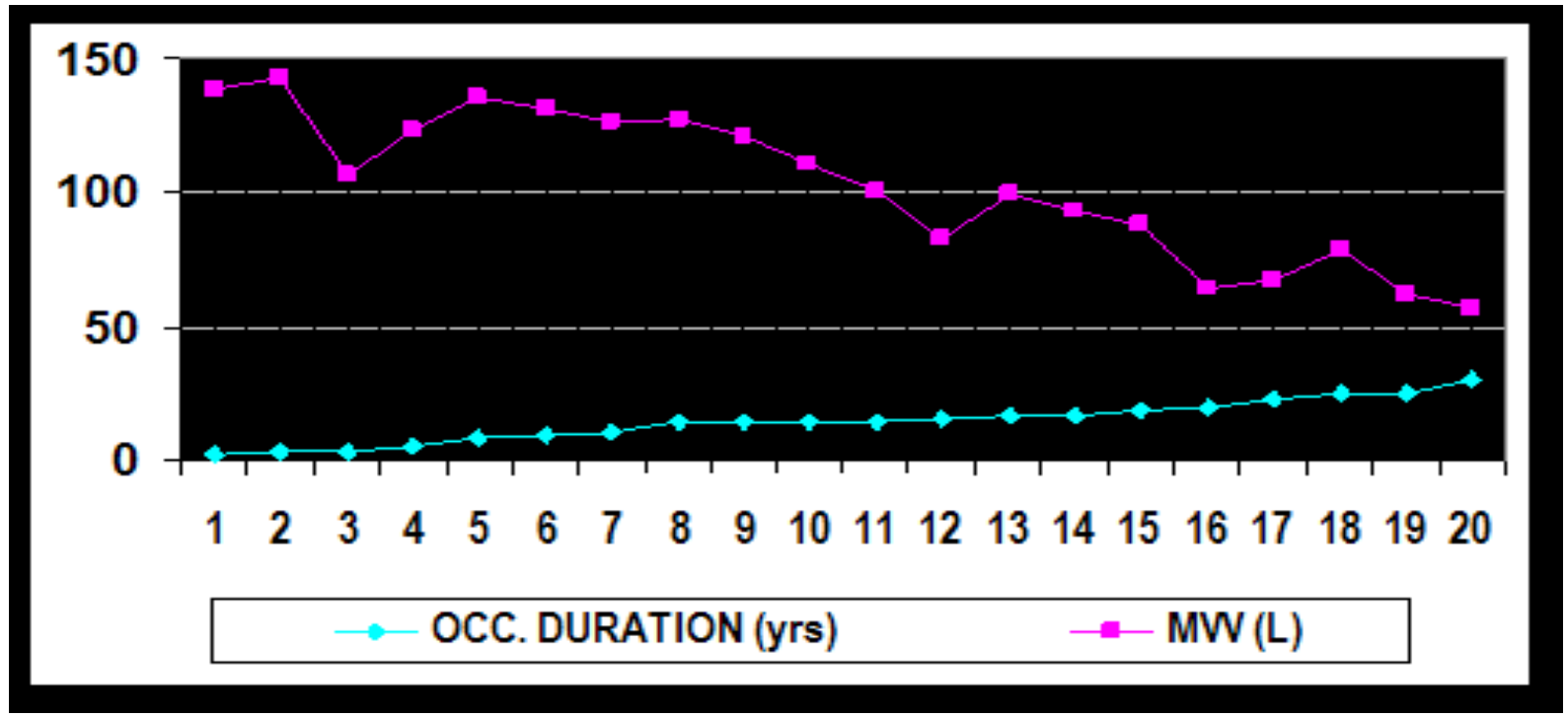

Fig 3: Shows the line graph comparison of MVV values with the occupational years

\section{Discussion}

Vellore and its surroundings has got many tannery workers where they are involved in various processes before the final production and exposing themselves to harmful chemicals. Chromium is one among the chemicals which can cause cancer of not only the lungs but also the bladder,kidney, nasal cavity. ${ }^{(9)}$ Many studies have been done to assess the effect of such harmful chemicals in lung functions. Long term exposure to these biological hazards like endotoxins mycotoxins could be another cause of reduction in lung function because during the tanning process, the hide contains numerous microorganisms which serve as a medium for exposure. In another study showed that chronic and long term exposure to tanning chemicals can cause asthma related symptoms that is features of obstructive lung disease. This study was done among tannery workers working in Tuzla Organized Industry Region, Istanbul was doneto know whether long term exposure to chemical affects lung function parameters showed a significant deficit in the lung parameters of $\mathrm{FVC} \%, \mathrm{FEV}_{1} \%, \mathrm{FEV}_{1} / \mathrm{FVC} \%$, $\mathrm{PEF} \%, \mathrm{FEF}_{25-75 \%}{ }^{(10)}$ All the lung parameters were also reduced in another study done in Kanpur leather where the study group showed the features of bronchial obstruction. $^{(8)}$

Our study too showed moderate restriction and mild obstruction when compared to normal subjects. This study correlates with previous study of tannery workers and chrome plate workers. It also shows progressive decrement in FVC, FEFmax and MVV with their age and duration of occupation. Decrement in MVV which was found may be due to aging process. Respiratory illness was found in $60 \%$ of our study group when compared to $<40 \%$ in control group.

Long-term exposure to biological hazards, especially to microbial agents; endotoxins, proteases and mycotoxins, about which there is a large literature, may be another reason for continuing complaints. During the tanning process, risk of exposure to biological agents is ever-present, since the hide serves as a medium for numerous microorganisms

Long-term exposure to biological hazards, especially to microbial agents; endotoxins, proteases and mycotoxins, about which there is a large literature, may be another reason for continuing complaints. During the tanning process, risk of exposure to biological agents is ever-present, since the hide serves as a medium for numerous microorganisms

Long-term exposure to biological hazards, especially to microbial agents; endotoxins, proteases and mycotoxins, about which there is a large literature, may 
be another reason for continuing complaints. During the tanning process, risk of exposure to biological agents is ever-present, since the hide serves as a medium for numerous microorganisms

Long-term exposure to biological hazards, especially to microbial agents; endotoxins, proteases and mycotoxins, about which there is a large literature, may be another reason for continuing complaints. During the tanning process, risk of exposure to biological agents is ever-present, since the hide serves as a medium for numerous microorganisms

\section{Conclusion}

There is moderate restriction and mild obstruction in the study group when compared to the normal subjects and also shows progressive decrement in FVC as well as FEFmax with their age and duration of occupation in the tannery factories. The study recommends breathing exercises to improve the detoriated lung functions. The study can further be extended in near future by the monitoring in addition the diffusion capacities.

Acknowledgements: We sincerely thank our Dean, Government Vellore Medical College, Vellore for support and help to do this work. We thank the faculties of the department of Physiology and Tannery workers and volunteers for the control group for their full cooperation for our study.

Ethical Committee Clearance: Institutional Ethical committee clearance obtained.

Conflict of Interest: No conflict of interest applicable for this study.

\section{Source of Funding: Self}

\section{References}

1. "Activities and schemes operated by District Industries Centre, Vellore district". Vellore District Administration. Retrieved 7 July 2013.
2. Stern FB. Mortality among chrome leather tannery workers and update. Am J Ind Med. 2003Aug;44(2):197-206.

3. Lin SC, Tai CC. Nasal septum lesion caused by chromium among chromium electroplating workers. Am J Ind Med. 1994;26:221-8.

4. Basketter D, Horev L, Slodovnik D, Merimes S, Trattner A, Ingber A. Investigation of the threshold for allergic reactivity to chromium. Contact Dermatitis. 2001;44:70-4.

5. Budhwar R, Das M, Bihari V, Kumar S. Exposure estimates of chrome platters in Indian exploratory study. Biomarkers. 2005;10:252-7.

6. International Agency for Research on Cancer (IARC). IARC monographs on the evaluation of carcinogenic risks to humans. Leather tanning and processing. WHO: Lyon; 1987;7:25.

7. Mikoczy Z, Hagmar L. Cancer incidence in the Swedish leather tanning industry: Updated findings 1958-99. Occup Environ Med. 2005;62(4):61-4.

8. Occupational health risks among the workers occupational health risks among the workers employed in leather tanneries at Kanpur employed. Indian Journal of Occupational and Environmental Medicine. 2008 December; 12(3):32-35.

9. Kornhauser C, Katarzyna W, Kazimierz W, Malacara JM, Laura EN, Gomez L,et al. Possible adverse effect of chromium in occupational exposure of tannery workers. Industrial Health. 2002;40:207-13.

10. Issever H, Ozdillil K Ozyilidirium BA, Hapicioglu $\mathrm{b}$, Ince $\mathrm{H}$, et at. Respiratory problems in tannery workers in Istanbul. Indoor Built Environ 2007;16:177-83. 